\title{
Data Compression Applied to Electric Power Quality Tracking of ARC Furnace Load
}

\section{Chi-Jui Wu}

Professor, Department of Electrical Engineering, National Taiwan University of Science and Technology, Taipei, Taiwan, R.O.C. Professor, Department of Electrical Engineering, National Taiwan Marine University, Keelung, Taiwan, R.O.C.

\section{Tsu-Hsun Fu}

Associate Professor, Department of Electrical Engineering, Chin-Min College, Miaoli, Taiwan, R.O.C.

Follow this and additional works at: https://jmstt.ntou.edu.tw/journal

Dart of the Electrical and Computer Engineering Commons

\section{Recommended Citation}

Wu, Chi-Jui and Fu, Tsu-Hsun (2003) "Data Compression Applied to Electric Power Quality Tracking of ARC Furnace Load," Journal of Marine Science and Technology. Vol. 11: Iss. 1, Article 5.

DOI: $10.51400 / 2709-6998.2277$

Available at: https://jmstt.ntou.edu.tw/journal/vol11/iss1/5

This Research Article is brought to you for free and open access by Journal of Marine Science and Technology. It has been accepted for inclusion in Journal of Marine Science and Technology by an authorized editor of Journal of Marine Science and Technology. 
Data Compression Applied to Electric Power Quality Tracking of ARC Furnace Load

Acknowledgements

This work was supported in part by the National Science Council of R.O.C under Grant NSC 90-2213-E011-064. 


\title{
DATA COMPRESSION APPLIED TO ELECTRIC POWER QUALITY TRACKING OF ARC FURNACE LOAD
}

\author{
Chi-Jui $\mathrm{Wu}^{*}$ and Tsu-Hsun $\mathrm{Fu}^{* *}$
}

Key words: electric power quality, data compression, discrete wavelet transform, multi-resolution analysis, coding.

\begin{abstract}
The data compression techniques are used to enhance the storage capability of electric power quality instruments in monitoring distorted waveforms of electric arc furnaces. Both voltage flicker in voltage waveforms and harmonic components in current waveforms are major disturbances. The compression approaches by multiresolution analysis developed from the discrete wavelet transform with threshold coding and vector quantization coding are compared. The results from spectrum analysis show high compression ratios while keep low information loss. The error levels between original and reconstructed waveforms are discussed. Field measured waveforms from arc furnace loads are used to show the goodness of the data compression techniques. From the calculation results, the reconstructed voltage waveforms using threshold coding almost keep the same voltage flicker values. However, the vector quantization coding is better for harmonic distorted current waveforms. Thereafter, in the practical application of data compression for arc furnace loads, the threshold coding is used for recording voltage waveforms, and vector quantization coding for current waveforms. For the field measurement voltage and current waveforms, this method can reduce data memory size to $15.11 \%$ and $24.51 \%$, respectively, while keep power quality characteristics. This paper provides a good approach to longtime electric power quality tracking of disturbing loads.
\end{abstract}

\section{INTRODUCTION}

Fluctuating loads, such as electric arc furnaces and electric welders, may cause the disturbances of voltage flicker (voltage fluctuation) in a weak power system and affect the illumination in nearby distribution area [5]. By the way, not only the DC arc furnaces, but also the $\mathrm{AC}$ arc furnaces will produce harmonic currents. Both

Paper Submitted 12/26/02, Accepted 04/01/03. Author for Correspondence: Chi-Jui Wu.

*Professor, Department of Electrical Engineering, National Taiwan University of Science and Technology, Taipei, Taiwan, R.O.C.

Professor, Department of Electrical Engineering, National Taiwan Marine University, Keelung, Taiwan, R.O.C.

**Associate Professor, Department of Electrical Engineering, Chin-Min College, Miaoli, Taiwan, R.O.C. voltage flicker and harmonic currents are major power quality disturbances caused by arc furnaces. In evaluation and limitation, there are some definitions and standards to quantify the disturbance levels, such as IEEE 519-1992 [8] and IEC 61000 [6, 7]. The total harmonic distortion (THD), short-term voltage flicker severity (Pst), and long-term voltage flicker severity (Plt) are used. However, sometimes it is desired to record voltage and current waveforms in the specified duration to track the disturbance levels. The instantaneous waveforms are helpful for detailed analysis both in limitation and mitigation. However, in the recording of instantaneous voltage and current waveforms with disturbances, a few seconds original signal could need the memory of several mega bytes. Then, under the consideration of memory and cost, the data recording length of an instrument will be limited. How to effectively reduce data size becomes a typical issue.

There are basically many approaches to compress data size. While lossless data compression wants to keep the signal information, lossy data compression gives better compression ability considering acceptable errors. The later could use suitable transforms and coding techniques. A general condition is that the data are analyzed by a transform and therefore a coding method is to extract the characteristic information and remove redundancy. Many transform methods have been used, such as discrete Fourier transform, shortterm discrete Fourier transform, discrete cosine transform (DCT) [16], and discrete wavelet transform (DWT) $[10,16]$. With respective to coding, the entropy coding has been used in most compression standards such as JPEG [2] and MPEG [1]. There are still several representatives of the state-of-art coders, such as embedded zero-tree DCT coding (EZDCT), embedded zero-tree wavelet coding (EZW), zero-tree entropy coding (ZTE), set partition in hierarchical trees (SPIHT) coding, embedded block coding with optimized truncation (EBCOT) [20], and vector quantization (VQ) [4, 16, 18].

By preserving feature integrity and removing redundancy to obtain a higher compression result, the 
DWT is a good way to preprocess data compression. With the same compression ratio, the DWT provides a less reconstruction error than the DCT [10]. By the time-frequency domain characteristics, it is effective in localizing analysis of time-sequence sampled data. The DWT has been used in the power quality measurement to effectively detect the exact time of disturbances and track signal fluctuation, such as harmonics and voltage flicker $[11,12,14]$. The DWT is also a feasible way to compress the data in power quality measurement $[3,10$, $13,14,15,19]$.

In this paper, the DWT with threshold and vector quantization coding is used to enhance the storage capacity in recording waveforms of arc furnace loads where voltage flicker and harmonic currents are major disturbances. The application of the DWT in multiresolution analysis (MRA) is to decompose the original signal in several levels. The approaches of threshold coding and vector quantization coding are used to extract the characteristic information and remove the redundancy. The compression ratio $(\mathrm{CR})$ and normalized mean-square error (NMSE) are used to reveal the goodness of compression techniques. The differences of voltage flicker $10-\mathrm{Hz}$ equivalent values and harmonic distortion factors between original waveforms and reconstructed waveforms could be used to show that this method is helpful in voltage flicker and harmonic current tracking.

\section{WAVELET TRANSFORM}

The definition of the continuous wavelet transform of signal $\boldsymbol{x}(\boldsymbol{t})$ is

$$
\begin{aligned}
& \boldsymbol{C W T}(\boldsymbol{a}, \boldsymbol{b})=\left\langle\boldsymbol{x}(\boldsymbol{t}), \psi_{a, b}(t)\right\rangle=\int_{-\infty}^{+\infty} \boldsymbol{x}(\boldsymbol{t}) \psi_{a, b}(t) d t \\
& \psi_{a, b}(t)=\psi((t-b) / a) / \sqrt{a}
\end{aligned}
$$

where $\boldsymbol{a}$ is the scale parameter, and $\boldsymbol{b}$ is the time shift parameter. The wavelet basic function $\psi_{\boldsymbol{a}, \boldsymbol{b}}(\boldsymbol{t})$ is defined on the right half plane of $(\boldsymbol{a}, \boldsymbol{b})$, that is, $(\boldsymbol{b} \in \boldsymbol{R}, \boldsymbol{a}>0)$. The original signal $\boldsymbol{x}(\boldsymbol{t})$ can be reconstructed by the parameter $(\boldsymbol{a}, \boldsymbol{b})$. The inverse continuous wavelet transform is given by

$$
x(t)=\frac{1}{C_{\psi}} \int_{-\infty}^{+\infty} \int_{-\infty}^{+\infty} C W T(a, b) \psi_{a, b}(t) \frac{d a d b}{a^{2}}
$$

where $\boldsymbol{C}_{\psi}$ is the normalized constant.

For a digital recorder with a sampling period $T$, the discrete wavelet transform (DWT) is needed. Selecting $\boldsymbol{a}=\boldsymbol{a}_{0}^{\boldsymbol{m}}, \boldsymbol{b}=\boldsymbol{n} \boldsymbol{a}_{0}^{\boldsymbol{m}} \boldsymbol{b}_{0}, \boldsymbol{t}=\boldsymbol{k} \boldsymbol{T}$, and $k, m, n \in Z$, the DWT is

$$
=\sum_{k} x[k] \psi^{*}\left[\left(\boldsymbol{k}-\boldsymbol{n} \boldsymbol{a}_{0}^{\boldsymbol{m}} \boldsymbol{b}_{0}\right) / \boldsymbol{a}_{0}^{\boldsymbol{m}}\right.
$$

The inverse discrete wavelet transform (IDWT) is given by

$$
\boldsymbol{x}[\boldsymbol{k}]=\left(\sum_{m} \sum_{n} \boldsymbol{D} \boldsymbol{W} \boldsymbol{T}(\boldsymbol{m}, \boldsymbol{n}) \psi^{*}\left[\left(\boldsymbol{k}-\boldsymbol{n} \boldsymbol{a}_{0}^{m} \boldsymbol{b}_{0}\right) / \boldsymbol{a}_{0}^{m}\right) / \boldsymbol{C}_{\psi}\right.
$$

Equation (4) could be said to an FIR filter with as the pulse response of the digital filter. So we can use multi-resolution filters to achieve DWT or IDWT with different resolution levels [3].

\section{DATA COMPRESSING AND CODING}

The purpose of data compression is to reduce the number of data for expressing signal information by getting rid of duplicated data. The basic concept is described in Fig. 1, where $\boldsymbol{C}_{0}[\boldsymbol{n}]$ is the original signal (measured voltage and current waveforms) and $\hat{\boldsymbol{C}}_{0}[\boldsymbol{n}]$ is the reconstructed signal (reconstructed voltage and current waveforms): The process of compression gives encoded data $\boldsymbol{C}_{0}[\boldsymbol{n}]$ (storaged voltage and current waveforms), and therefore, the process of reconstruction uses encoded data. Different encoding techniques can result in different compression effect. The threshold $(\mathrm{TH})$ coding and vector quantization (VQ) coding will be used and compared.

\section{Threshold (TH) coding}

If the DWT is applied to data compressing, it will relate to the wavelet set address characteristics in multiresolution analysis (MRA). A threshold analysis is used as the simple coding method to reduce unimportant information. Fig. 2 shows the flow chart of a 3-level MRA decomposition and reconstruction method, where $\boldsymbol{H}[\boldsymbol{n}]$ and $\boldsymbol{G}[\boldsymbol{n}]$ are a high-pass filter and a low-pass filter, respectively. The circular blocks mean downsamplers and up-samplers, respectively. $\boldsymbol{C}_{\boldsymbol{s}-1}[\boldsymbol{n}]$ is resolved into $\boldsymbol{C}_{s}[\boldsymbol{n}]$ and $\boldsymbol{D}_{s}[\boldsymbol{n}], s=1,2$, and 3, where the high frequency band coefficients $\boldsymbol{D}_{\boldsymbol{s}}[\boldsymbol{n}]$ will be processed through a threshold coding. The output-sequence values are given by

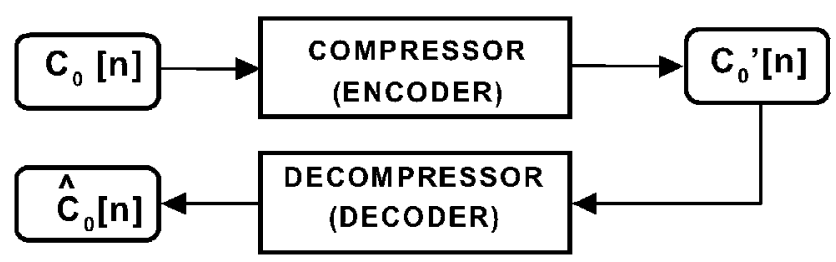

Fig. 1. Data compressing system block diagram.

$$
\boldsymbol{D W T}(\boldsymbol{m}, \boldsymbol{n})=\left\langle\boldsymbol{x}[\boldsymbol{k}], \psi_{m, n}[k]\right\rangle
$$




$$
\hat{\boldsymbol{D}}_{s}[\boldsymbol{n}]=\left\{\begin{array}{cc}
\boldsymbol{D}_{s}[\boldsymbol{n}] & \left|\boldsymbol{D}_{s}[\boldsymbol{n}]\right| \geq \eta_{s} \\
0 & \left|\boldsymbol{D}_{s}[\boldsymbol{n}]\right|<\eta_{s}
\end{array}\right.
$$

Using this simple threshold method, the decomposition data in $\boldsymbol{D}_{s}[\boldsymbol{n}]$ with absolute values less than $\eta_{s}$ are regarded as noises and filtered out. Therefore, the stored data for reconstruction are non-zero parts from $\boldsymbol{D}_{s}[\boldsymbol{n}]$, addresses of data, and $\boldsymbol{C}_{s}[\boldsymbol{n}]$.

The next step is how to select a threshold value. The simplest method at the $s^{\text {th }}$ resolution level is

$$
\eta_{s}=\boldsymbol{u} \times \max \left\{\left|\boldsymbol{D}_{s}[\boldsymbol{n}]\right|\right\}
$$

where $u$ is the threshold factor, and $0 \leq u \leq 1$. If $u=$ 0.05 , the threshold value $\eta_{s}$ becomes $5 \%$ of the maximal absolute value of the sequence $\boldsymbol{D}_{s}[\boldsymbol{n}]$. However, if there are transient components with very high amplitude in the original waveforms, this method will induce high error. To improve this problem, the average of absolute values of $\boldsymbol{D}_{\boldsymbol{s}}[\boldsymbol{n}]$ can be used. The threshold value in this paper will be

$$
\eta_{s}=K \times u \times \text { average }\left\{\left|D_{s}[n]\right|\right\}
$$

where $K$ is the magnification factor.

In order to reveal the efficiency of data compression, the compression ratio (CR) is defined as

$$
C R=\frac{\text { original file size }}{\text { compressed file size }}
$$

The evaluation of the signal quality after reconstruction can be achieved by the normalized meansquare error (NMSE), which is by the norm as

$$
\text { NMSE }=\frac{\left\|\boldsymbol{C}_{0}[n]-\hat{\boldsymbol{C}}_{0}[n]\right\|^{2}}{\left\|\boldsymbol{C}_{0}[n]\right\|^{2}}
$$

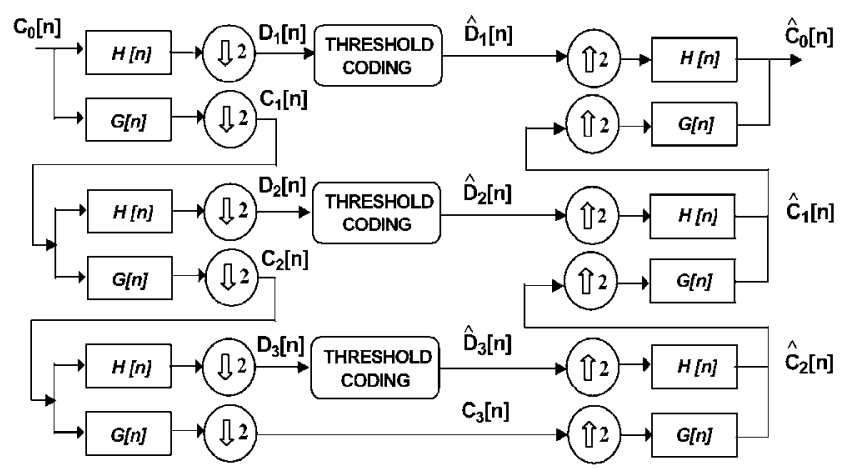

Fig. 2. Decomposition and reconstruction using 3-level multi-resolution analysis and threshold (TH) coding.
A lower NMSE means a smaller error between the reconstructed signal and the original signal.

\section{Vector Quantization (VQ) coding $[4,16,18]$}

This method divides the original signal into pieces and treats them as $n \times 1$ vectors. The typical samples to describe piece signals are named the codevectors or codewords $\boldsymbol{Y}_{j}$ where $j=1,2, \ldots, M$. The set of codevectors contribute to a codebook. The codevector $\boldsymbol{Y}_{\boldsymbol{k}}$ to represent a piece signal $\boldsymbol{X}$ is chosen from the codebook, which makes $d\left(\boldsymbol{X}, \boldsymbol{Y}_{k}\right) \leq \boldsymbol{d}\left(\boldsymbol{X}, \boldsymbol{Y}_{j}\right)$ for $j=1,2, \ldots, M$, where $\boldsymbol{d}\left(\boldsymbol{X}, \boldsymbol{Y}_{j}\right)$ represents the error between $\boldsymbol{X}$ and $\boldsymbol{Y}_{\boldsymbol{j}}$. The most popular method for evaluating error is using the square of Euclidean distance. Therefore

$$
\boldsymbol{d}\left(\boldsymbol{X}, \boldsymbol{Y}_{j}\right)=\left\|\boldsymbol{X}-\boldsymbol{Y}_{j}\right\|^{2}=\sum_{l=1}^{n}\left(\boldsymbol{x}_{l}-\boldsymbol{y}_{j l}\right)^{2}
$$

where $\boldsymbol{X}=\left(\boldsymbol{x}_{1}, \boldsymbol{x}_{2}, \ldots, \boldsymbol{x}_{\boldsymbol{n}}\right)^{t}$ and $\boldsymbol{Y}_{\boldsymbol{j}}=\left(\boldsymbol{y}_{j 1}, \boldsymbol{y}_{j 2}, \ldots, \boldsymbol{y}_{\boldsymbol{j}}\right)^{t}$. After choosing the codevector $\boldsymbol{Y}_{k}$, the index $k$ will be expressed using $\log _{2} M$ bits and sent out. At the decoding terminal, there exists the same codebook, which makes it possible to find $\boldsymbol{Y}_{\boldsymbol{k}}$ to represent $\boldsymbol{X}$ after receiving the index $k$.

The application of a 3-level MRA and VQ coding is shown in Fig. 3, where the size of codebook in each resolution level would be different. Therefore, a different size of codebook could result in different quantization effect, which means different compression result and reconstruction error. The most popular codebook design method is to use the Linde-Buzo-Gray (LBG) training [4, 9]. It is not described in details here. According to the wavelet subband theory, the lower frequency band coefficients may contain more information, and they are more important to reconstruct the signal. On the other hand, the higher frequency band coefficients contain less information and are less important. As a result, the size of codebook for the

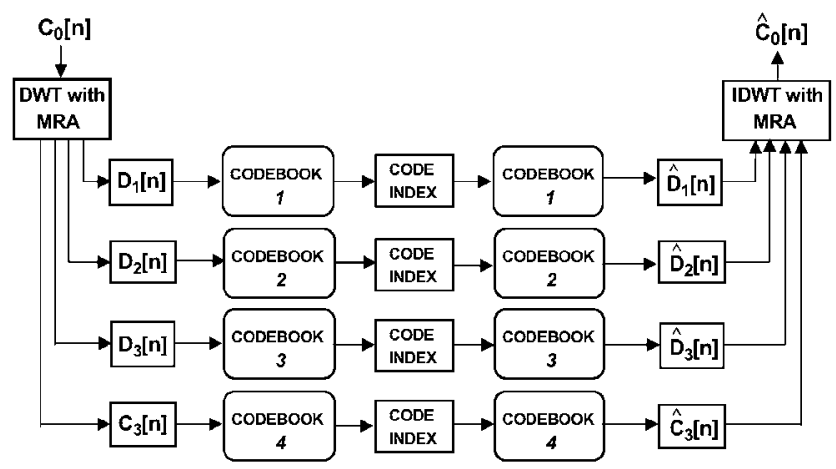

Fig. 3. Decomposition and reconstruction using 3-level multi-resolution analysis and vector quantization (VQ) coding. 
lower frequency band coefficients should be larger. For training the codebook in this paper, all codevectors are of one dimension. It also can be said a special scalar quantization.

\section{WAVEFORMS OF VOLTAGE FLICKER AND HARMONICS}

\section{Voltage flicker}

One of major disturbances from arc furnace loads is voltage flicker problem in the voltage waveforms. In a short duration, a voltage flicker waveform can be described as

$$
v(t)=\sqrt{2} V_{r m s}\left[1+\frac{1}{2} \sum_{n} \Delta V_{-n} \sin \left(2 \pi f_{n} t+\varphi_{n}\right)\right] \sin \left(2 \pi f_{s y s} t\right)
$$

where $f_{\text {sys }}$ is the fundamental frequency (power frequency, $60-\mathrm{Hz}$ ), $V_{r m s}$ is the RMS value of the voltage, $\Delta \boldsymbol{V}_{n}{ }_{n}$ is the amplitude modulation value of the modulation frequency $f_{n}$. For the voltage flicker limitation, we usually consider fn only in the range of $0.1 \mathrm{~Hz} \sim 30 \mathrm{~Hz}$. Fig. 4 shows a simple voltage flicker waveform, which contains only one modulation component, $\Delta \boldsymbol{V}_{-} 10=0.3$ pu and $f_{n}=10 \mathrm{~Hz}$.

The definitions of voltage deviation $\Delta \boldsymbol{V}$ and voltage flicker $10-\mathrm{Hz}$ equivalent value $\Delta \boldsymbol{V} 10$ are, respectively,

$$
\begin{aligned}
& \Delta V=\sqrt{\sum_{n}\left(\Delta V_{-n}\right)^{2}} \\
& \Delta V 10=\sqrt{\sum_{n}\left(a_{n} \Delta V_{-n}\right)^{2}}
\end{aligned}
$$

where $\boldsymbol{a}_{\boldsymbol{n}}$ is the flicker sensitivity coefficient of the frequency $f_{n}$ as shown in Fig. 5, that describes the sensitivity of mankind eyes-brain mechanism to illumi-

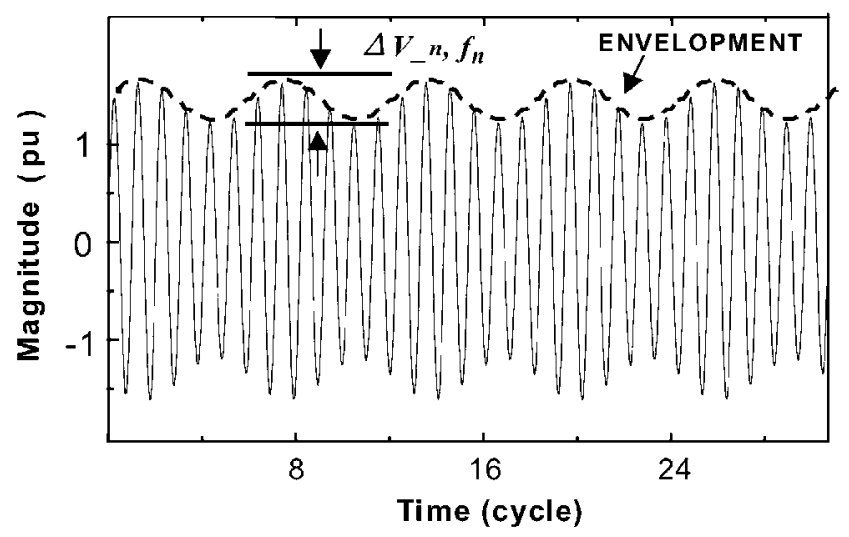

Fig. 4. A voltage flicker waveform, $\Delta V_{-} 10=0.3$ pu and $f_{n}=10 \mathrm{~Hz}$. nation flicker. The method with $\Delta \boldsymbol{V} 10$ is used by the Taiwan Power Company for voltage flicker limitation [17].

The first waveform $v$ in Fig. 6 is the given flicker waveform, whose five amplitude modulation components are given in the second row of Table 1. Because

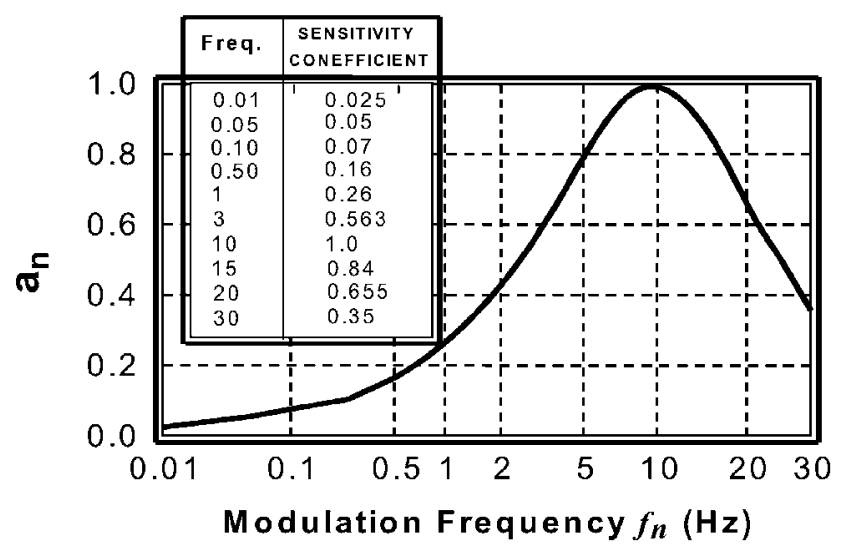

Fig. 5. Flicker sensitivity coefficient curve.
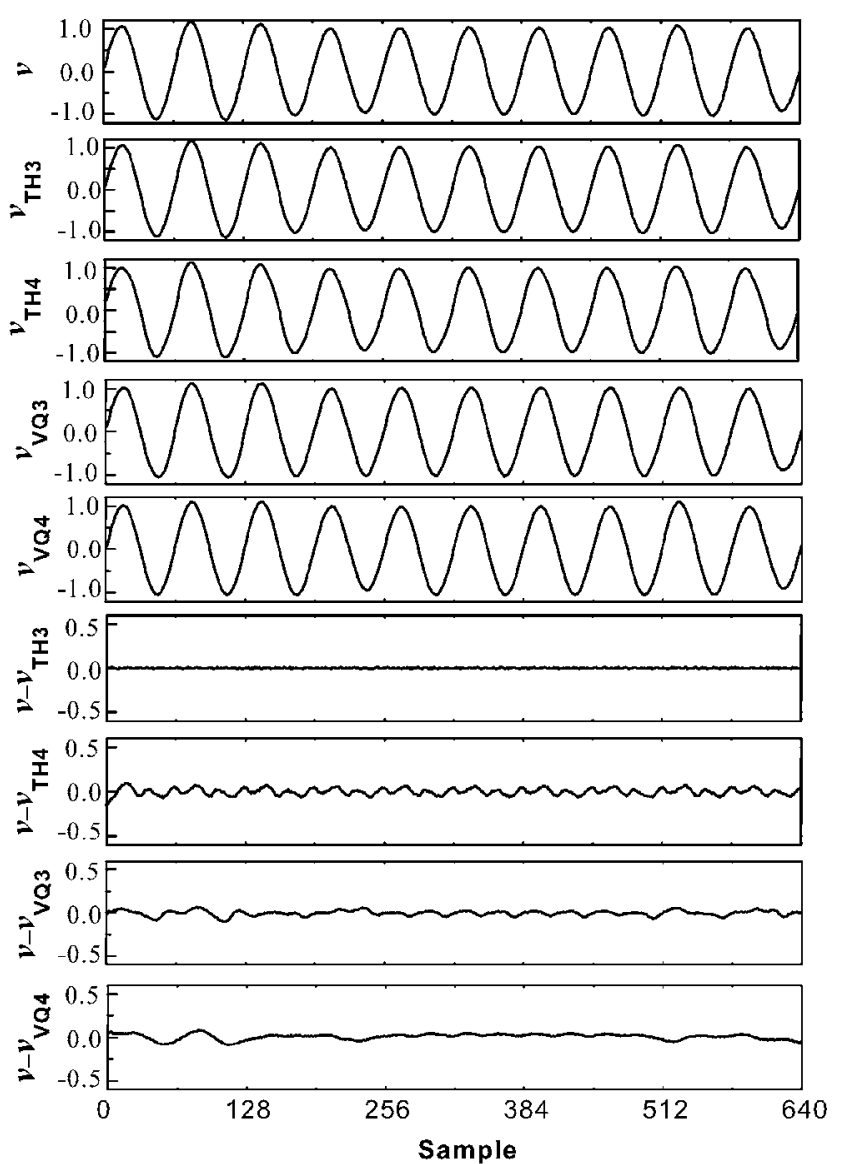

Fig. 6. Reconstruction results of a given voltage flicker waveform. 
Table 1. Compression results of the given Voltage flicker waveform

\begin{tabular}{|c|c|c|c|c|c|c|c|c|c|c|}
\hline & \multirow[t]{2}{*}{ Vrms } & \multicolumn{5}{|c|}{$\begin{array}{l}\text { Amplitude Modulation } \\
\text { Component }\end{array}$} & \multirow[t]{2}{*}{$\begin{array}{l}\Delta V \\
(\%)\end{array}$} & \multirow[t]{2}{*}{$\begin{array}{r}\Delta V 10 \\
(\%)\end{array}$} & \multirow[t]{2}{*}{ CR } & \multirow[t]{2}{*}{ NMSE } \\
\hline & & $\Delta V \_01$ & $\Delta V \_05$ & $\Delta V \_10$ & $\Delta V \_15$ & $\Delta V \_30$ & & & & \\
\hline Given Value & 1 & 0.031 & 0.052 & 0.08 & 0.047 & 0.003 & 11.08 & 9.85 & & \\
\hline TH2 & 1 & 0.031 & 0.052 & 0.080 & 0.047 & 0.003 & 11.08 & 9.85 & 3.9 & 5.13E-05 \\
\hline TH3 & 1 & 0.031 & 0.052 & 0.08 & 0.047 & 0.003 & 11.08 & 9.85 & 7.7 & 7.27E-05 \\
\hline TH4 & 1 & 0.030 & 0.05 & 0.077 & 0.045 & 0.003 & 10.66 & 9.47 & 14.6 & $2.18 \mathrm{E}-03$ \\
\hline TH5 & 1 & 0.028 & 0.047 & 0.073 & 0.042 & 0.003 & 10.05 & 8.94 & 26.7 & $1.18 \mathrm{E}-01$ \\
\hline VQ2 & 1 & 0.027 & 0.052 & 0.077 & 0.046 & 0.004 & 10.72 & 9.57 & 3.54 & 3.00E-04 \\
\hline VQ3 & 1 & 0.016 & 0.041 & 0.061 & 0.031 & 0.007 & 8.17 & 7.38 & 4.23 & $2.10 \mathrm{E}-03$ \\
\hline VQ4 & 1 & 0.019 & 0.028 & 0.044 & 0.020 & 0.010 & 5.98 & 5.22 & 4.70 & $3.55 \mathrm{E}-03$ \\
\hline VQ5 & 1 & 0.010 & 0.023 & 0.036 & 0.021 & 0.006 & 4.9 & 4.41 & 4.87 & $1.24 \mathrm{E}-02$ \\
\hline
\end{tabular}

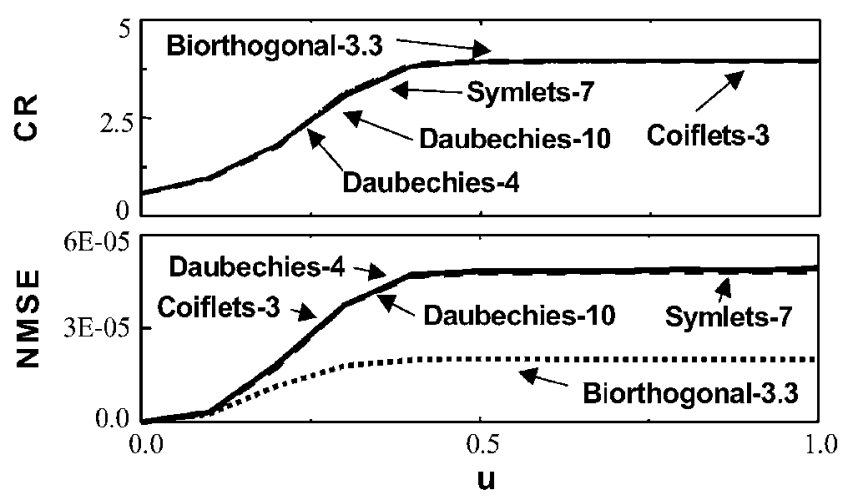

Fig. 7. Effect of threshold factor $u$ on CR and NMSE of a given voltage flicker waveform with different wavelet basic functions.

there are so many kinds of wavelet basic functions, only Daubechies-4, Daubechies-10, Biorthogonal-3.3, Symlets-7, and Coiflets-3 have been chosen for comparison. The compression results of this waveform using 2-level MRA with threshold coding $(K=8)$ and different wavelet basic functions are shown in Fig. 7. The CR and NMSE values are a little different. However, it has been evaluated but not shown here that the $\Delta \boldsymbol{V}$ and $\Delta \boldsymbol{V} 10$ values of the reconstructed waveforms using different wavelet basic functions are similar. With a lower NMSE and an acceptable CR, the Biorthogonal-3.3 with $u=0.5$ is used for the following study.

The compression results of the given voltage flicker waveform are shown in Table 1, where THs and VQs, $s$ $=2,3,4$, and 5, mean the s-level MRA method with threshold coding and VQ coding, respectively. The codebook sizes of high frequency band coefficients and low frequency band coefficients in each level are given in Appendix. With the threshold coding, the CR becomes higher with a higher decomposition level, but the
Table 2. Comparison of NMSE values with a same CR of the given voltage flicker waveform

\begin{tabular}{ccc}
\hline $\mathrm{CR}=4.23$ & $\mathrm{TH} 3(\mathrm{u}=0.284)$ & $\mathrm{VQ3}$ \\
\hline $\mathrm{NMSE}$ & $6.61 \mathrm{E}-05$ & $2.10 \mathrm{E}-03$ \\
\hline
\end{tabular}

error will be larger. With a same resolution level, the $\mathrm{CR}$ values with threshold coding are larger than that with the VQ coding. From the comparison of $\Delta \boldsymbol{V} 10$ and $\Delta \boldsymbol{V}$, the values from $\mathrm{TH} 2$ and $\mathrm{TH} 3$ are almost the same with the given values. But with the VQ coding, there may be significant errors in $\Delta \boldsymbol{V} 10$ and $\Delta \boldsymbol{V}$. With MRA method, using down sampling strategy, the higher the level is, the lower the cut off frequency becomes. Since low-frequency coefficients are major information, too much coefficients removing will cause high errors. So, the errors of TH5 and VQ5 are excessively high and they cannot be used. Fig. 6 also shows reconstruction results of the given voltage flicker waveform. The original waveform is $v$, the reconstructed waveforms obtained from TH3 and TH4 with threshold coding method are depicted as $\boldsymbol{v}_{\mathbf{T H} 3}$ and $\boldsymbol{v}_{\mathbf{T H} 4}$, respectively, and that with the VQ coding method as $v_{\mathrm{VQ} 3}$ and $\boldsymbol{v}_{\mathrm{VQ}}$, respectively. The following waveforms show the errors between the original and reconstructed waveforms. With the TH3, the error is negligible. The 3-level MRA method with threshold coding (TH3) would be suitable if the CR, NMSE, and accuracy of $\Delta \boldsymbol{V} 10$ and $\Delta \boldsymbol{V}$ are considered. Table 2 gives the comparison of NMSE values with a same CR of the given flicker waveform. Under the same CR condition, the threshold coding has a better compression result. From the results in Fig. 6, Table 1 and Table 2, it can be found that the threshold coding is better than the VQ coding for voltage flicker waveforms to effectively keep accuracy of voltage flicker values. 
Table 3. Compression results of the given harmonic current waveform

\begin{tabular}{|c|c|c|c|c|c|c|c|c|}
\hline & \multirow{2}{*}{$\begin{array}{l}\text { Fundamental } \\
\text { Value }\end{array}$} & \multicolumn{4}{|c|}{ Harmonic Components } & \multirow{2}{*}{$\begin{array}{l}\text { THD } \\
(\%)\end{array}$} & \multirow[t]{2}{*}{ CR } & \multirow[t]{2}{*}{ NMSE } \\
\hline & & $5^{\text {th }}$ & $7^{\text {th }}$ & $11^{\text {th }}$ & $13^{\text {th }}$ & & & \\
\hline Given Value & 1 & 0.1 & 0.05 & 0.03 & 0.02 & 11.75 & & \\
\hline TH2 & 1 & 0.103 & 0.038 & 0.019 & 0.009 & 11.18 & 2.4 & $1.14 \mathrm{E}-03$ \\
\hline TH3 & 1 & 0.095 & 0.034 & 0.019 & 0.008 & 10.30 & 2.81 & $1.57 \mathrm{E}-03$ \\
\hline TH4 & 1 & 0.092 & 0.035 & 0.019 & 0.009 & 10.07 & 2.91 & $4.79 \mathrm{E}-03$ \\
\hline TH5 & 1 & 0.091 & 0.037 & 0.019 & 0.009 & 10.05 & 3.17 & $1.21 \mathrm{E}-01$ \\
\hline VQ2 & 1 & 0.104 & 0.052 & 0.026 & 0.017 & 12.04 & 3.46 & 2.07E-04 \\
\hline VQ3 & 1 & 0.105 & 0.05 & 0.026 & 0.017 & 12.04 & 4.08 & $1.97 \mathrm{E}-04$ \\
\hline VQ4 & 1 & 0.106 & 0.051 & 0.027 & 0.018 & 12.12 & 4.49 & 4.33E-04 \\
\hline VQ5 & 1 & 0.109 & 0.055 & 0.029 & 0.019 & 12.65 & 4.63 & $9.22 \mathrm{E}-03$ \\
\hline
\end{tabular}
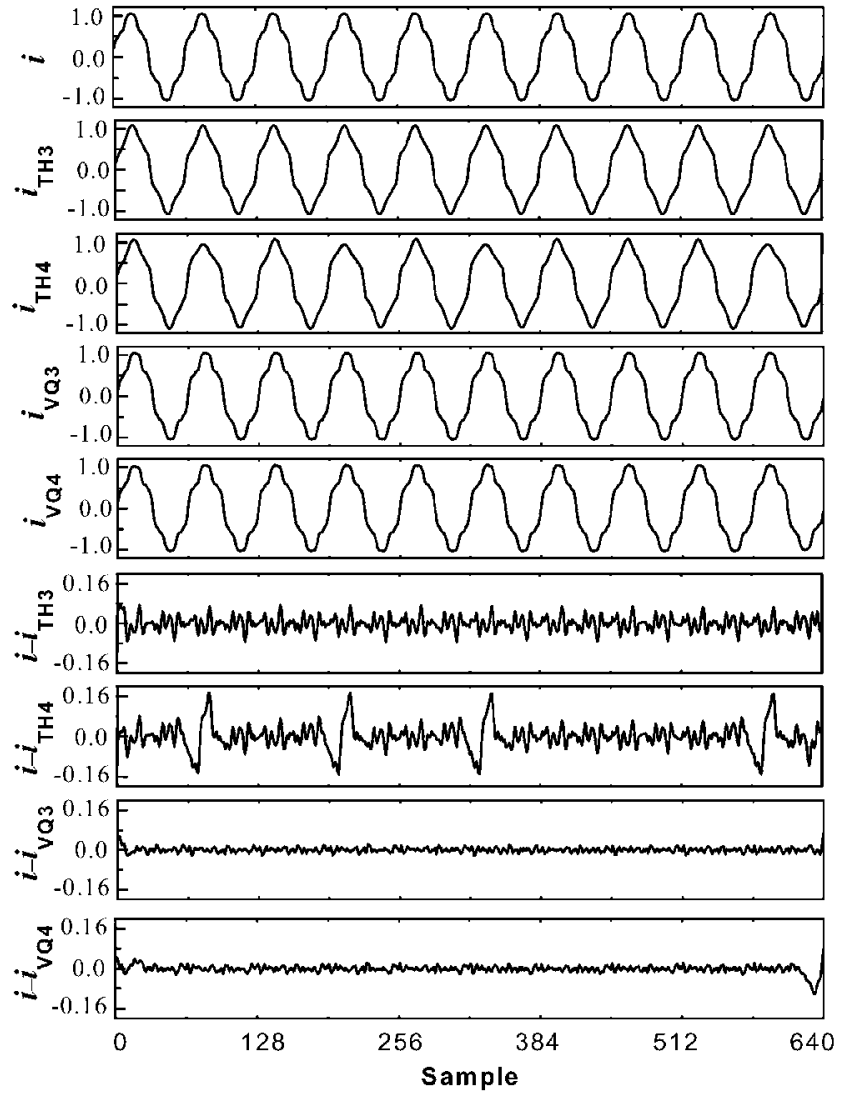

Fig. 8. Reconstruction results of a harmonic distorted current waveform.

\section{Harmonics}

The other major disturbance from arc furnace loads is the harmonic components in current waveforms. At steady state, a harmonic distorted current waveformcan be expressed as
Table 4. Comparison of NMSE values with a same CR of the given harmonic current waveform

\begin{tabular}{ccc}
\hline $\mathrm{CR}=4.08$ & $\mathrm{TH} 3(\mathrm{u}=0.6)$ & $\mathrm{VQ3}$ \\
\hline NMSE & $2.33 \mathrm{E}-03$ & $1.97 \mathrm{E}-04$ \\
\hline & \\
$\boldsymbol{i}(\boldsymbol{t})=\boldsymbol{I}_{0}+\sqrt{2} \boldsymbol{I}_{1} \sin \left(\omega_{1} \boldsymbol{t}+\theta_{1}\right)+\sum_{h=2}^{\infty} \sqrt{2} \boldsymbol{I}_{h} \sin \left(\boldsymbol{h} \omega_{1} \boldsymbol{t}+\theta_{h}\right)$
\end{tabular}

where $\boldsymbol{I}_{0}=\mathrm{dc}$ component, $\boldsymbol{I}_{1}=$ fundamental RMS component, $\omega_{1}=$ fundamental angular frequency, and $h=$ harmonic order. The first part of Fig. 8 reveals the given harmonic distorted current waveform with four harmonic components given in the second row of Table 3 . The other parts in Fig. 8 give the reconstructed waveforms and errors, where $\boldsymbol{i}_{\mathbf{T H} 3}$ and $\boldsymbol{i}_{\mathbf{T H} 4}$ mean with threshold coding under 3 and 4-level MRA. And $\boldsymbol{i}_{\mathrm{VQ} 3}$ and $\boldsymbol{i}_{\mathrm{VQ} 4}$ mean with VQ coding. Table 3 shows the results up to 5 -level resolution of the given harmonic current waveform. Where magnification factor $K$ is 5 . The reconstruction results are relatively correct up to the 3level resolution with the VQ coding. But, the errors with the threshold coding are larger. Table 4 shows the comparison of NMSE values with a same CR of the given harmonic current waveform. Under the same CR condition, the VQ coding has an obviously better compression result. From the results in Fig. 8, Table 3 and Table 4, it can be found that the VQ coding is better than the threshold coding for harmonic distorted current waveforms.

\section{APPLICATION TO FIELD MEASUREMENT WAVEFORMS}

In order to understand furthermore the effect of threshold coding and VQ coding in practical signals, we 
Table 5. Compression results of field measured voltage flicker and harmonic current waveforms

\begin{tabular}{|c|c|c|c|c|c|c|c|}
\hline & \multicolumn{4}{|c|}{$\begin{array}{c}\text { Voltage Flicker } \\
\text { Waveform }\end{array}$} & \multicolumn{3}{|c|}{$\begin{array}{c}\text { Harmonic Current } \\
\text { Waveform }\end{array}$} \\
\hline & $\begin{array}{l}\text { DV } \\
(\%)\end{array}$ & $\begin{array}{c}\text { DV10 } \\
(\%)\end{array}$ & $\mathrm{CR}$ & NMSE & $\begin{array}{l}\text { THD } \\
(\%)\end{array}$ & $\mathrm{CR}$ & NMSE \\
\hline True Values & 6.21 & 4.49 & & & 12.04 & & \\
\hline TH3 & 6.25 & 4.5 & 6.62 & $1.36 \mathrm{E}-03$ & 11.13 & 1.37 & $3.64 \mathrm{E}-03$ \\
\hline TH4 & 6.92 & 4.98 & 11.22 & $4.47 \mathrm{E}-03$ & 11.12 & 1.40 & $3.91 \mathrm{E}-03$ \\
\hline VQ3 & 7.0 & 4.98 & 4.23 & $2.02 \mathrm{E}-03$ & 12.01 & 4.08 & $1.38 \mathrm{E}-03$ \\
\hline VQ4 & 8.48 & 5.76 & 4.7 & $6.15 \mathrm{E}-03$ & 12.22 & 4.49 & $3.85 \mathrm{E}-03$ \\
\hline
\end{tabular}

$(\mathrm{kV})$
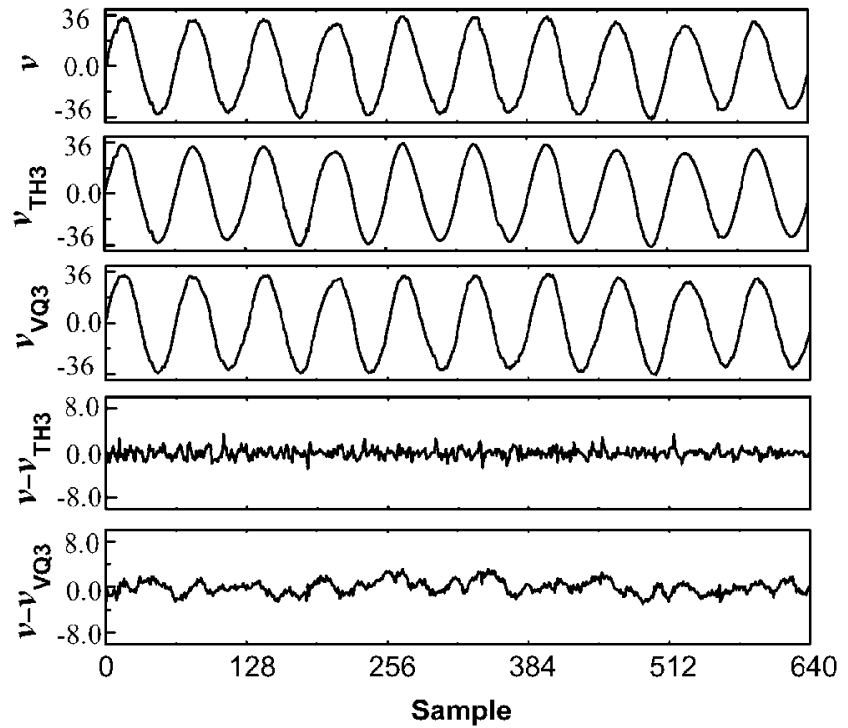

Fig. 9. Reconstruction results of a measurement voltage flicker waveform.

adopt measured voltage waveforms of a steel-making factory with an arc furnace. The voltage waveform in Fig. 9 has obvious voltage flicker. The results of data compression are given in Table 5. The $K$ values for voltage flicker waveforms and harmonic waveforms are 7.5 and 2.6, respectively in threshold coding. The CRs of 3-level MRA with threshold coding and VQ coding, respectively, are 6.62 and 4.23 , and the NMSE are $1.36 \mathrm{E}-03$ and $2.02 \mathrm{E}-03$. With TH3, the errors in $\Delta \boldsymbol{V} 10$ and $\Delta \boldsymbol{V}$ are negligible. Fig. 10 shows amplitude modulation components in the reconstructed waveform. There is almost no difference using the threshold coding.

Fig. 11 shows the harmonic distorted current waveform of the arc furnace where rectifier circuits are used. Table 5 gives the compression results. The CRs are 1.37 and 4.08, respectively, using TH3 and VQ3, and NMSEs are 3.64E-03 and 1.38E-03, respectively. With VQ3, the error in THD is negligible. Fig. 12 shows

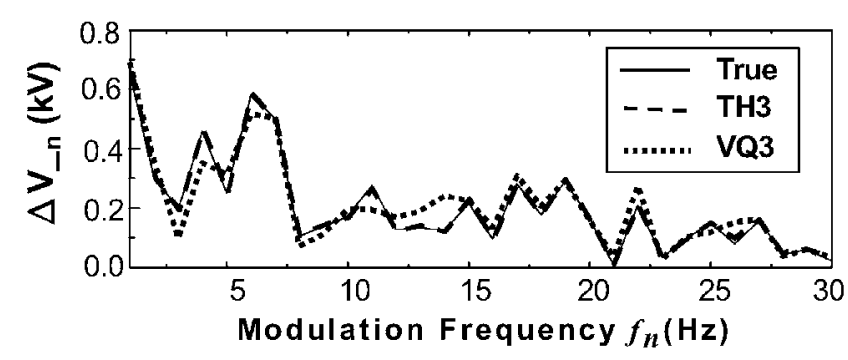

Fig. 10. Comparison of threshold coding and vector quantization coding on flicker amplitude modulation components in reconstructed voltage flicker waveform (3-level MRA).

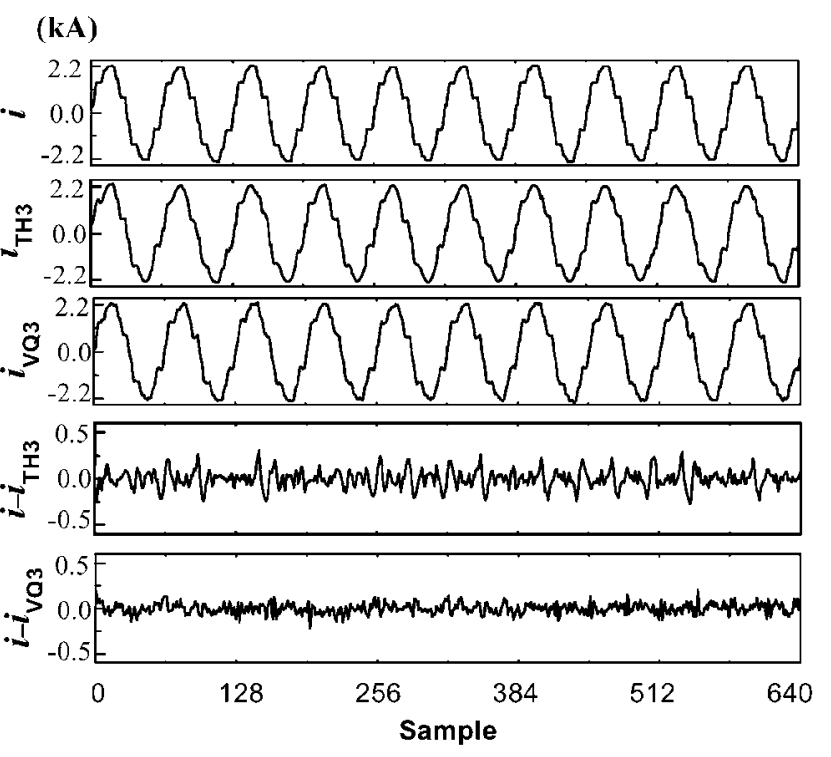

Fig. 11. Reconstruction results of a measurement harmonic current waveform.

harmonic current distributions in the reconstructed waveform. There are acceptable errors using VQ3.

It also can be concluded that while the VQ coding is suitable for the harmonic distorted current waveform, the threshold coding is better for the voltage flicker waveform. If the TH3 is used for the voltage waveform 


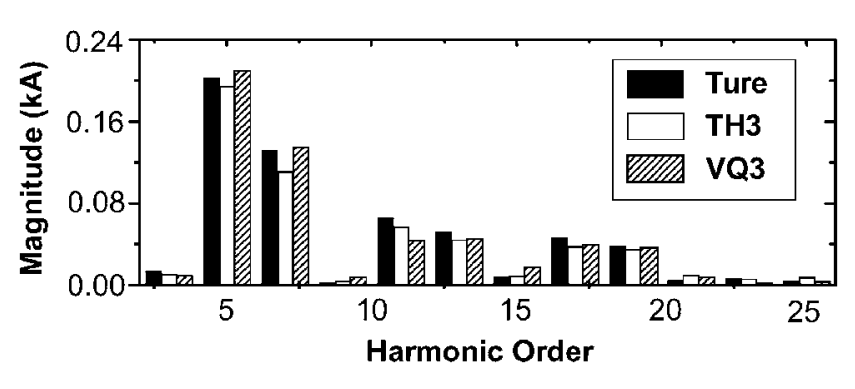

Fig. 12. Comparison of threshold coding and vector quantization coding on harmonic components in reconstructed harmonic current waveform (3-level MRA).

and VQ3 for the current waveform, the CR is 6.62 and 4.08 , respectively. As a result, the data memory size can be reduced to $15.11 \%$ and $24.51 \%$ respectively. This approach can provide a good method for long-time power quality tracking.

\section{CONCLUSION}

The data compression methods using discrete wavelet transform have been presented to be effective in reducing the data storage size of waveforms of arc furnace loads with voltage flicker and harmonics distortions. In the recording of arc furnace data, the vector quantization coding is suitable for current waveforms to keep harmonic information, and for voltage waveforms, it is better to use the threshold coding, because the errors in voltage flicker values are small. Although a high level multi-resolution analysis can give a larger compression ratio, the error also becomes larger. There should be a compromise. It is better to use the differences of voltage flicker values and harmonic distortion factors between original waveforms and reconstructed waveforms as the error index. This is useful in the design of power quality instruments.

\section{ACKNOWLEDGEMENT}

This work was supported in part by the National Science Council of R.O.C under Grant NSC 90-2213-E011-064.

\section{REFERENCES}

1. Chiariglione, L., "MPEG and Multimedia Communications," IEEE Trans. on Circuits and Systems for Video Technology, Vol. 7, pp. 5-18 (1997).

2. Christopoulos, C., Skodras, A.N., and Ebrahimi, T., "The JPEG2000 Still Image Coding System: An Overview," IEEE Trans. on Consumer Electronics, Vol. 46, pp. 1103-1127 (2000).
3. Gaouda, A.M., Salama, M.M.A., Sultan, M.R., and Chikhani, A.Y., "Power Quality Detection and Classification Using Wavelet Multi-resolution Signal Decomposition," IEEE Trans. on Power Delivery, Vol. 14, pp. 1469-1476 (1999).

4. Haber, J. and Seidel, H.P., "Using an Enhanced LBG Algorithm to Reduced the Codebook Error in Vector Quantization," Proc. Computer Graphics International, pp. 99-104 (2000).

5. Heydt, G.T., Electric Power Quality, Stars in a Circle Publications, Indiana, U.S.A. (1994).

6. IEC, Limitation of Voltage Fluctuations and Flicker in Low-Voltage Power Supply Systems for Equipment with Rated Current $\leq 16$ A, IEC 61000-3-3, Electromagnetic Compatibility (EMC), Part 3: Limits-Section 3, Swiss (1994).

7. IEC, Limitation of Voltage Fluctuations and Flicker in Low-Voltage Power Supply Systems for Equipment with Rated Current Greater Than 16A, IEC 61000-3-5, Electromagnetic Compatibility (EMC), Part 3: LimitsSection 5, Swiss (1994).

8. IEEE, IEEE Recommended Practices and Requirements for Harmonic Control in Electrical Power System, IEEE Standard 519-1992, New Jersey, U.S.A. (1993).

9. Linde, Y., Buzo, A., and Gray, R., "An Algorithm for Vector Quantizer Design," IEEE Trans. on Communications, Vol. 28, pp. 84-95 (1980).

10. Little, T.B. and Morrow, D.J. "Wavelets for the Analysis Compression of Power System Disturbances," IEEE Trans. on Power Delivery, Vo1. 14, pp. 358-364 (1999).

11. Poisson, O., Rioual, P. and Meunier, M., "New Signal Processing Tools Applied to Power Quality Analysis," IEEE Trans. on Power Delivery, Vol. 14, pp.561-566 (1999).

12. Robertson, D.C., Camps, O.I., Mayer, J.S., and Gish, W. B., "Wavelets and Electromagnetic Power System Transients," IEEE Trans. on Power Delivery, Vol. 11, pp.1050-1058 (1996).

13. Santoso, S., Grady, W.M., Powers, E.J., Lamoree, J., and Bhatt, S.C., "Characterization of Distribution Power Quality Events with Fourier and Wavelet Transforms," IEEE Trans. on Power Delivery, Vol. 15, pp. 247-254 (2000).

14. Santoso, S., Powers, E.J., and Grady, W.M., "Power Quality Disturbance Data Compression Using Wavelet Transform Methods," IEEE Trans. on Power Delivery, Vol. 12, pp.1250-1257 (1997).

15. Santoso, S., Powers, E.J., Grady, W.M., and Hofmann, P., "Power Quality Assessment Via Wavelet Transform Analysis," IEEE Trans. on Power Delivery, Vol. 11, pp. 924-930 (1996).

16. Sayood, K., Introduction to Data Compression, $2^{\text {nd }}$ ed., Morgan Kaufmann, San Francisco, U.S.A. (2000).

17. Taipower, Voltage Flicker Temporary Limitation 
Standard, Taiwan Power Company, Taipei, Taiwan, ROC (1995).

18. Wang, X., Wu, G., and Lin, X., "Wavelet Image Coding with Lattice Vector Quantization," Proc. $3^{\text {rd }}$ International Conference on Signal Processing, Vol. 2, pp. 966969 (1996).

19. Wilkinson, W.A. and Cox, M.D., "Discrete Wavelet Analysis of Power System Transients," IEEE Trans. on Power Systems, Vol. 11, pp. 2038-2044 (1996).

20. Zhao, D., Chan, Y.K., and Gao, W., "Low-Complexity and Low-Memory Entropy Coder for Image Compression," IEEE Trans. on Circuits and Systems for Video Technology, Vol. 11, pp. 1140-1145 (2001).

\section{APPENDIX}

The codebook sizes of VQ coding in different MRA levels are given in Table A.

Table A. Codebook sizes

\begin{tabular}{ccc}
\hline Level $s$ & $D_{s}$ & $C_{s}$ \\
\hline$s=1$ & 2 & 64 \\
$s=2$ & 4 & 32 \\
$s=3$ & 8 & 16 \\
$s=4$ & 4 & 8 \\
$s=5$ & 2 & 8 \\
\hline
\end{tabular}

